\title{
Disfagia en tiempos de COVID-19
}

\section{Dysphagia in COVID-19 times}

\author{
Lara Fernández R. ${ }^{1,2}$, Natalia Cabrera S. ${ }^{1,3}$, Diego Fernández O. ${ }^{1}$, Lorena Olcese $T^{1}$
}

\section{Resumen}

Los pacientes con COVID-19 pueden presentar dificultades en la alimentación por distintos factores, entre los que destacan el aumento del esfuerzo respiratorio, uso de dispositivos de apoyo ventilatorio, compromiso neurológico y disfagia postextubación. La evaluación clínica es fundamental, pero debe ser realizada con precaución y con elementos de protección personal, ya que es un procedimiento generador de aerosoles, al igual que la evaluación instrumental mediante videofluoroscopía y evaluación fibroendoscópica de la deglución. Las recomendaciones de manejo deben basarse en la evaluación clínica adaptada, tanto para pacientes ambulatorios como hospitalizados, y debe incluir el manejo nutricional, compensatorio y el seguimiento clínico periódico para evitar las consecuencias de la disfagia orofaríngea, y así disminuir la tasa de neumonía aspirativa, causa importante de morbimortalidad.

Palabras clave: disfagia, deglución, COVID-19, SARS-CoV-2.

\begin{abstract}
Patients with COVID-19 may present feeding difficulties due to different factors, like the increase in respiratory effort, use of ventilatory support devices, neurological compromise and post-extubation dysphagia. Clinical evaluation is essential, but it must be carried out with caution and using personal protection elements, since it is an aerosol-generating procedure, as well as the instrumental evaluation by videofluoroscopy and fiberoptic endoscopic evaluation of swallowing. Treatment should be based on adapted clinical evaluation, for both outpatients and hospitalized patients, and should include nutritional treatment, compensatory management and periodic clinical follow-up to avoid the consequences of oropharyngeal dysphagia and decrease the rate of aspiration pneumonia major cause of morbidity and mortality.
\end{abstract}

Keywords: dysphagia, swallowing, COVID-19, SARS-CoV-2.

'Clínica Santa María. Santiago,
Chile.
${ }^{2}$ Hospital Clínico Universidad
de Chile. Santiago, Chile.
${ }^{3}$ Hospital El Carmen de Maipú.
Santiago, Chile.
Los autores declaran no tener
conflicto de interés.
Recibido el 16 de julio de
2020. Aceptado el 20 de julio
de 2020.
Correspondencia:
Natalia Cabrera S.
Av. Santa María 0500,
Providencia. Santiago, Chile.
Email: ncabrera.soto@gmail.
com

\section{Introducción}

La disfagia orofaríngea (DO) tiene una prevalencia en la población general que varía entre $2,3 \%$ y $16 \%^{1}$ y aumenta con la edad, siendo cercana al $30 \%$ en adultos mayores independientes ${ }^{2,3}, \mathrm{y}$ con las enfermedades neurológicas, cerebrovasculares y degenerativas ${ }^{4}$. Si se evalúan pacientes que han sido hospitalizados en unidades de cuidados intensivos y han necesitado ventilación mecánica invasiva, se ha encontrado una incidencia variable de disfagia postextubación que va de un $3 \%$ a $62 \%$, y de estos pacientes un $60 \%$ persiste con DO al alta hospitalaria ${ }^{5}$ La importancia de la
DO radica en que un porcentaje importante de estos pacientes tendrá malnutrición, deshidratación y presentará neumonías por aspiración, aumentando su morbimortalidad y afectando su calidad de $v i a^{6-8}$. Múltiples estudios avalan que la sola presencia de DO aumenta la estancia hospitalaria, los reingresos, costos médicos totales y la institucionalización ${ }^{9-11}$.

La enfermedad por coronavirus (COVID-19), causada por coronavirus tipo 2 asociado al síndrome respiratorio agudo grave (SARS$\mathrm{CoV}-2$ ), ha tenido un crecimiento exponencial expandiéndose por todos los continentes. En Chile ha afectado a pacientes de todas las edades, siendo el 15,9\% mayores de 60 años, 
con una tasa de hospitalización por cuadros graves que llega a ser 7 veces mayor que en pacientes menores de 60 años ${ }^{12}$. Además, los pacientes que han requerido hospitalización tienen factores de riesgo cardiovasculares y patologías pulmonares crónicas que inciden tanto en la gravedad de la infección viral, como en la vulnerabilidad del paciente para presentar DO y sus complicaciones ${ }^{13}$. Dentro de las manifestaciones de la enfermedad se incluye el compromiso respiratorio, trombosis microvascular y disfunción neurológica ${ }^{14}$, lo que asociado a una hospitalización prolongada en una unidad de cuidados intensivos incrementa el riesgo de presentar disfagia.

Los equipos multidisciplinarios encargados de la evaluación y tratamiento de la disfagia deben lidiar con los casos habituales de DO no asociados a COVID-19, con los pacientes que tienen disfagia asociada a la infección viral y con los pacientes que tenían DO y además presentaron COVID-19. En esta revisión de la literatura, se discutirá sobre la fisiopatología, evaluación y tratamiento de la disfagia orofaríngea en el contexto de COVID-19, para así sugerir recomendaciones de manejo de esta condición.

\section{Fisiopatología: Afección multinivel}

En una deglución intervienen más de 30 pares de músculos, un aparato osteocartilaginoso extenso y diversos nervios craneales que llevan las aferencias sensitivas de las estructuras mencionadas y traen las eferencias para una respuesta motora orofaríngea adecuada. La deglución está coordinada por una extensa red neuronal que incorpora estructuras corticales, subcorticales y del tronco encefálico. Los pacientes con COVID-19 pueden presentar disfagia por distintos factores que afecten esta red neural y sus efectores. Se han descrito alteraciones neurológicas en un tercio de los pacientes hospitalizados con COVID-19. Las alteraciones del sistema nervioso central incluyen vértigo, cefalea, compromiso de conciencia, accidentes cerebrovasculares, encefalitis, ataxia y convulsiones. Las afecciones del sistema nervioso periférico incluyen alteraciones del olfato y el gusto, alteraciones visuales, dolor neuropático y síndrome de Guillain-Barré ${ }^{15}$. Asociado a ello, en pacientes intubados, hospitalizados en unidades de cuidados intensivos y con patologías graves existen tres causas importantes que pueden conllevar a DO: el diagnóstico en sí, las comorbilidades previas y el tratamiento en unidad de pacientes críticos, que puede producir disfagia postextubación (DPE). La disfagia postextubación es consecuencia del trauma orofaríngeo y/o laríngeo (secundario al tubo endotraqueal, sonda nasoenteral, succión traqueal, entre otros), neuromiopatía del paciente crítico, reflujo gastroesofágico, compromiso de conciencia (por la patología y/o sedación), reducción de la sensibilidad faringolaríngea, disincronía respiración/deglución como consecuencia de la ventilación mecánica y, eventualmente, compresión del nervio laríngeo recurrente por el balón del tubo endotraqueal; lo anterior reduce las habilidades del paciente para manejar sus secreciones, proteger la vía aérea y deglutir ${ }^{16}$; produciendo aspiración silente postextubación hasta en un $25 \%$ de los pacientes ${ }^{17,18}$, aumentando aún más la morbimortalidad. Sin mencionar que la higiene oral en algunas oportunidades queda relegada a un segundo plano olvidando considerar que la placa bacteriana es uno de los factores de riesgo más relevantes para presentar neumonías aspirativas y asociadas a ventilación mecánica ${ }^{19}$. Además, la posición prono puede ocultar una mala higiene oral y dificultar su manejo. Por otro lado, la vía aerodigestiva superior, como su nombre lo indica, es un tubo que permite la deglución y la respiración de una forma coordinada. Cuando respiramos por la nariz el esfínter velopalatino y el laríngeo están abiertos para permitir el paso del aire, pero cuando deglutimos ambos se cierran para proteger la vía aérea. El momento preciso en que ocurre cada evento y su coordinación nos permite deglutir principalmente en la fase posinspiratoria o espiratoria temprana, seguido de una breve apnea y, finalmente, la espiración. En pacientes con patologías respiratorias y/o postventilación mecánica, incluidos los pacientes con COVID-19, existe una descoordinación respiración-deglución. En estos casos existen degluciones durante la inspiración aumentando el riesgo de aspiración y exacerbaciones en patologías pulmonares crónicas ${ }^{20}$.

Finalmente, una vez recuperados del periodo agudo de la infección viral, se han reportado 
consecuencias en el periodo subagudo como debilidad muscular, fatiga, rigidez articular, problemas neuropsicológicos y del funcionamiento en términos de movilidad, actividades de la vida diaria y disfagia ${ }^{21}$.

En un estudio realizado en Brasil por Santilli de Lima y cols., se incluyeron 101 pacientes con COVID-19 que fueron intubados una media de $8,8 \pm 8,1$ días. A las $24 \mathrm{~h}$ de su extubación se realizó una evaluación clínica en la cabecera implementando el protocolo $d y s$ phagia risk evaluation. El 18\% de los pacientes requirieron vía alternativa de alimentación y el 53\% tuvieron deglución segura, pero requirieron medidas compensatorias ${ }^{22}$.

\section{Evaluación y tratamiento de la disfagia orofaríngea}

La evaluación de la DO es compleja porque compromete un amplio espectro de intervenciones como son la evaluación clínica, la evaluación instrumental mediante videofluoroscopía (VFC) o evaluación de deglución con videoendoscopio flexible (FEES por su sigla en inglés), la evaluación de la higiene oral y evaluación nutricional. Ello requiere que el paciente con DO deba ser evaluado por un equipo interdisciplinario, lo que muchas veces es complejo, porque esta patología ocurre en contextos diversos como son las unidades de cuidados intensivos, unidades de hospitalización general y de rehabilitación, así como en ambientes ambulatorios y comunitarios.

Una vez que tenemos un diagnóstico preciso, necesitamos tratar a ese paciente a través de medidas compensatorias, de rehabilitación o modificadoras de la enfermedad, donde se involucra un espectro mayor de actores y de intervenciones. Durante una pandemia, todo este enfoque se ve afectado por la disponibilidad de recursos clínicos y humanos, y por la factibilidad de realizar dichas intervenciones con el menor riesgo posible para los pacientes y los profesionales sanitarios. Dado que SARSCoV-2 es un virus nuevo, no contamos con la evidencia científica suficiente para realizar revisiones sistemáticas y metaanálisis y generar recomendaciones del manejo y tratamiento de la disfagia en pacientes con COVID-19 en base a ello. La literatura aumenta y cambia rápidamente. Sin embargo, sabemos algunos aspectos técnicos de la transmisión viral y contamos con recomendaciones de diversas entidades internacionales en las cuales basaremos las nuestras.

\section{Recomendaciones generales}

En el contexto de la pandemia actual, todos los pacientes son teóricamente considerados como contagiados. La conducta del profesional que evalúa al paciente debe ser cautelosa debido al porcentaje desconocido de pacientes contagiados que son asintomáticos y porque los test nasofaríngeos evaluados mediante la técnica de reacción de polimerasa en cadena (PCR) nasofaríngea tienen cerca de un 30\% de falsos negativos ${ }^{23,24}$.

La Organización Mundial de la Salud (OMS) concluyó que las principales vías de transmisión son el contacto y las gotas respiratorias $^{25}$. Cuando un paciente tose o estornuda, se genera un gas turbulento que lleva millones de gotas de distintos tamaños. Según su tamaño, la turbulencia y la velocidad de la nube de gas se pueden ir asentando o evaporando. La humedad y temperatura local permite que algunas de ellas, consideradas como aerosoles, permanezcan suspendidas en el aire por horas y puedan generar contagios por vía inhalatoria. Esta nube puede viajar hasta 7-8 metros y las gotas que vayan cayendo contaminan superficies $^{26}$.

Muchos pacientes con COVID-19 y disfagia están predispuestos a toser durante las evaluaciones y terapias. La tos refleja que puede generarse durante la evaluación (ya sea durante el examen físico, por contacto con instrumental o por aspiración de saliva, alimentos o líquidos) puede ser prolongada y difícil de suprimir, potenciando la diseminación de pequeñas gotas. Además, durante la evaluación se querrá conocer la fuerza de la tos voluntaria como mecanismo de protección de la vía aérea, por lo que de todos modos se estará expuesto a un mecanismo potente de transmisibilidad y esto es independiente de que un paciente con COVID-19 puede tener tos basalmente por el compromiso de su vía aérea. Por todo ello se considera que tanto la evaluación como el tratamiento de la disfagia producen aeroso$\operatorname{les}^{27,28} \mathrm{y}$, por ende, se deben tomar las medidas de precaución correspondientes que incluyen 
el uso adecuado de elementos de protección personal: mascarilla FFP2 o superior, protección facial completa, guantes, gorro y delantal desechable ${ }^{29,30}$.

Distintas sociedades han recomendado posponer todas las evaluaciones prescindibles en pacientes con COVID-19 hasta que se sospeche que el paciente deje de ser contagioso ${ }^{29}$. Algunas de las evaluaciones clínicas podrían realizarse a través de una teleconsulta. Sin embargo, existe un porcentaje de pacientes que debemos atender presencialmente. Conociendo los riesgos de retrasar el tratamiento de un paciente con disfagia, la evaluación y las indicaciones terapéuticas deben ser oportunas. Dada la propensión de aspiración silente post-extubación, la evaluación de la disfagia es crítica en un paciente con un estatus pulmonar frágil durante el transcurso o la recuperación de COVID- $19^{30}$.

\section{Recomendaciones para la evaluación clínica}

\subsection{Pacientes ambulatorios}

$\mathrm{La}$ anamnesis de un paciente con DO puede hacerse por teléfono o videollamada. Idealmente se sugiere contar con familiares o cuidadores para enriquecer los datos aportados. La percepción del paciente sobre sus síntomas puede obtenerse al aplicar escalas como la eating assessment tool-10 (EAT-10), validada al español ${ }^{31,32}$. Mediante esta vía también puede evaluarse la cognición y comunicación y su estatus de ingesta actual con la escala functional outcome swallowing scale (FOSS) ${ }^{33}$ o similar. Parte del examen físico puede obtenerse por videollamada si el paciente o su cuidador logran comprender bien las indicaciones y tenemos una buena calidad de imagen: postura, cierre labial, movimientos linguales, calidad vocal, esfuerzo respiratorio, fuerza tusígena, intentos para limpiar secreciones y un examen parcial de pares craneanos.

En casos de disfagia no severa, donde el paciente y algún miembro de la familia o cuidador puedan cooperar y cuenten con espesantes, podría realizarse un test de deglución de volumen-viscosidad (V-VST por sus siglas en inglés) ${ }^{34}$ evaluando cambios en la voz y tos como signos de alteración de la seguridad y la presencia de deglución fraccionada, sello labial insuficiente y residuo oral y faríngeo (reportado por el paciente) como signos de alteración de la eficacia. Otros alimentos podrían adaptarse a las consistencias evaluadas en este test como un pudding, flan o yogurt. Si el paciente contara con un saturómetro, podríamos agregar la medición de la desaturación como signo de alteración de la seguridad.

Con la anamnesis, examen físico y este test, podríamos dar recomendaciones iniciales de medidas compensatorias en su ingesta. Si el paciente no puede ser evaluado por telemedicina o en la anamnesis se objetivan síntomas y signos de disfagia orofaríngea con riesgo de aspiración, se sugiere acudir a una evaluación presencial con fonoaudiólogo(a) u otorrinolaringólogo(a) especialistas en deglución.

\subsection{Pacientes hospitalizados}

La evaluación clínica al lado de la cama del paciente es fundamental ya que, probablemente, será la base de las indicaciones de ingesta. También es importante considerar que, de forma complementaria a la evaluación de la deglución, se debe realizar una evaluación nutricional y de la higiene oral que son de igual forma relevantes para prevenir la neumonía aspirativa. Esta evaluación sólo debe realizarse en pacientes que se encuentren vigiles, cooperadores, con situación respiratoria estable $(\mathrm{PaFi}$ óptima, sin uso de ventilación no invasiva ni mascarilla de alto flujo) y capaces de deglutir su propia saliva. El paciente puede permanecer con naricera de oxígeno durante la evaluación $^{35}$. Se debe posicionar al paciente sentado $\left(90^{\circ}\right)$ o máximo en $60^{\circ}$ de reclinación, y verificar que se encuentre con el pulsioxímetro conectado y funcionando para monitorear la saturación de oxígeno. Previamente a ingresar a la habitación del paciente se deben obtener datos relevantes de la anamnesis, tales como: - Edad.

- Comorbilidades previas que puedan ser factor de riesgo de severidad de COVID-19 o que se asocien con disfagia (enfermedades neurológicas, respiratorias, esofágicas, entre otras).

- Antecedentes de disfagia previa y/o de neumonía aspirativa.

- Diagnóstico de ingreso actual: Neumonía por SARS-CoV-2, y otros que pudiesen ser concomitantes, como accidente cerebro 
vascular y otras patologías neurológicas que también son factor de riesgo de disfagia.

- Intubación y/o traqueostomía durante la hospitalización. Detallar características y número de días de permanencia de cada uno.

- Vía de alimentación actual: Vía oral, enteral (sonda nasoenteral o gastrostomía) o parenteral.

- Síntomas y signos de disfagia durante o posterior a la ingesta, tales como: tos, carraspera, cambios en la voz, atragantamiento, sensación de residuo oral o faríngeo, alteración de selle labial, odinofagia o desaturación $>3 \%$ del basal. Si el paciente se encuentra en condiciones, se le puede entregar previamente la encuesta EAT-10 validada al español para cribar la presencia de disfagia.

- Neumonía aspirativa durante la hospitalización.

Al entrar en la habitación del paciente se deben usar adecuadamente los elementos de protección personal, tomando en consideración protección para aerosoles ${ }^{27,28}$, y portando los instrumentos necesarios para realizar el V-VST ${ }^{35}$. Primero, observar al paciente y brevemente preguntar por los síntomas de disfagia ya detallados. Luego realizar un examen físico acotado, enfocándose principalmente en la evaluación de postura, simetría facial, selle labial, movimientos linguales, deglución en seco y saturación de oxígeno basal.

A continuación, si es necesario, realizar V-VST acortado ${ }^{36}$ con el objetivo de disminuir el tiempo de exposición y con ello de contagio (tanto para el paciente como para el evaluador). Este test consiste en un método abreviado del test de volumen-viscosidad clásico ${ }^{34}$ que explora los mismos signos de alteración de la seguridad (tos, cambios en la voz y desaturación $>3 \%$ ) y eficacia de la deglución (sello labial insuficiente, deglución fraccionada y sensación de residuo oral y/o faríngeo), con el fin de poder seleccionar el volumen y viscosidad del bolo óptimos para cada paciente con DO. La diferencia con el V-VST clásico es que solo se utiliza un volumen intermedio de bolo que corresponde a $10 \mathrm{~mL}$. El método (Figura 1) se inicia con $10 \mathrm{~mL}$ de consistencia néctar (viscosidad entre 250-300 $\mathrm{mPa} \cdot \mathrm{s}$ ), luego

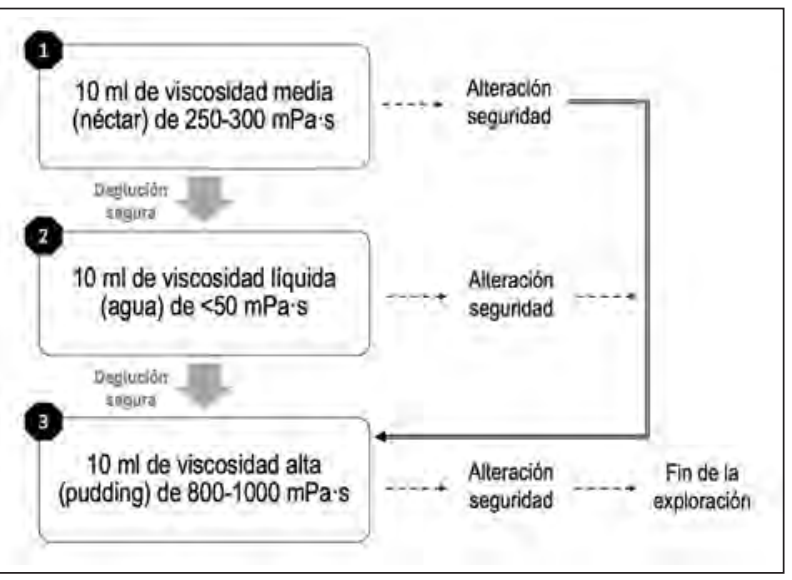

Figura 1. Test de volumen viscosidad acortado. Adaptado de Clavé $2008^{34}$ y $2020^{36}$.

con $10 \mathrm{~mL}$ de consistencia líquida (viscosidad $<50 \mathrm{mPa} \cdot \mathrm{s}$ ) y finaliza con $10 \mathrm{~mL}$ de consistencia pudding (viscosidad ideal entre 800-1.000 $\mathrm{mPa} \cdot \mathrm{s})$. Si el paciente presenta alguna alteración de la seguridad, la prueba se detiene y se procede a realizar la evaluación con la siguiente viscosidad más segura. Es importante tomar nota de las alteraciones producidas con cada consistencia (Tabla 1) para poder entregar una recomendación adecuada para la ingesta y para realizar el seguimiento durante la evolución, ya que este test puede ser repetido las veces que sea necesario durante la estancia hospitalaria y posterior al alta ${ }^{35}$.

Los pacientes con traqueostomía y COVID-19 deben usar cánula con balón y no fenestrada, y la evaluación clínica de la deglución debe ser realizada con el balón inflado para minimizar la tos y la producción de aerosoles ${ }^{35}$. Además, en estos pacientes se puede complementar la evaluación con el uso de colorante alimentario para mejorar el diagnóstico de aspiración, con un test de tinción azul de Evans modificado ${ }^{37}$. La evaluación finaliza con el diagnóstico clínico de disfagia y su severidad, que idealmente debe estar estandarizado por alguna escala validada para estos fines, como la escala FOSS ${ }^{33}$. En este punto se debe realizar la valoración del riesgo/beneficio de solicitar una evaluación instrumental para certificar o complementar el diagnóstico, lo cual debe ser discutido en equipo multidisciplinario, teniendo en cuenta las recomendaciones discutidas en el siguiente punto. 
Tabla 1. Alteraciones de la seguridad y eficacia detectadas en test de volumen viscosidad acortado

$\begin{array}{lccc}\text { Viscosidad } & 250 \mathrm{mPa} \cdot \mathrm{s} \text { (néctar) } & \text { Líquido } & 800 \mathrm{mPa} \cdot \mathrm{s} \text { (pudding) } \\ \text { Volumen } & 10 \mathrm{~mL} & 10 \mathrm{~mL} & 10 \mathrm{~mL}\end{array}$

Alteraciones de la seguridad

\begin{tabular}{|l|l|l|l|}
\hline Cambios en la voz & & & \\
\hline Tos & & & \\
\hline Desaturación $>3 \%$ & & & \\
\hline
\end{tabular}

Alteraciones de la eficacia

\begin{tabular}{|l|l|l|l|}
\hline Selle labial & & & \\
\hline Residuo oral & & & \\
\hline Deglución fraccionada & & & \\
\hline Residuo faríngeo & & & \\
\hline
\end{tabular}

En cada casilla en blanco se registra la presencia (+) o ausencia (-) de los signos de seguridad o eficacia enlistados a la izquierda, para cada una de las viscosidades administradas. Adaptado de Clavé $2020^{36}$.

\section{Recomendaciones para la evaluación instrumental}

La evaluación de la deglución mediante endoscopía flexible (FEES) no está recomendada de rutina dado que el paciente no puede usar mascarilla y existe un alto riesgo de proyectar gotas de pequeño tamaño a través de la tos y estornudos durante el examen ${ }^{29}$. Por otro lado, es un examen que no permite mantener una distancia adecuada y habitualmente considera la presencia de más de un profesional en la misma sala (otorrinolaringólogo(a), fonoaudiólogo(a), técnicos(as) en enfermería). Cuando se requiere realizar una FEES se deben balancear los riesgos y beneficios. Una alternativa sería acortar el protocolo de evaluación para disminuir el tiempo de exposición, pero de todos modos la nasofaringe contiene la mayor carga viral de la vía aerodigestiva superior en las etapas iniciales de la infección ${ }^{38}$.

Frente a esta situación, si se evidencia alto riesgo de aspiración durante la evaluación clínica, el examen preferido para la evaluación instrumental es la VFC. Excepciones a ello serían la presencia de disfonía, estridor o que el paciente no pueda ser movilizado a la sala de radiología. En estos casos se debe hacer una FEES con máxima precaución. Para limitar la aerosolización se sugiere no aplicar vasoconstrictores nasales en spray y el transporte de los nasofibroscopios debe ser en contenedores cerrados para minimizar el riesgo de transmisión por fomites ${ }^{30}$. En caso de realizarse una VFC considerar que el paciente se autoadministre los bolos con las diferentes consistencias para limitar el contacto con el paciente durante el examen y que al deglutir cada bolo se ponga una mascarilla para que, en caso de presentar tos en relación a la deglución, se limite la aerosolización ${ }^{30}$. Esto es difícil si además necesitamos mantener una postura adecuada durante la exposición.

\section{Recomendaciones de manejo}

\subsection{Manejo nutricional}

La estadía en unidad de paciente crítico es un factor de riesgo de malnutrición con pérdida de masa muscular y consecuente mayor morbimortalidad, por lo que la evaluación y manejo nutricional adecuado son de vital importancia en pacientes con COVID-19. La Sociedad Europea de Nutrición Clínica y Metabolismo (ESPEN) desarrolló en este contexto una guía práctica de manejo cuyas principales recomendaciones $\operatorname{son}^{39}$ :

- El cribado nutricional se debe considerar en los pacientes con COVID-19, en especial en población de mayor riesgo como adultos mayores y pacientes con patologías crónicas y multimorbilidades.

- Los pacientes con malnutrición deben ser tratados, considerando los ajustes de requerimientos calóricos, proteicos y de 
carbohidratos y grasas según factores de riesgo. Se deben emplear suplementos nutricionales orales cuando la ingesta vía oral de la dieta no cumpla con los requerimientos nutricionales.

- En pacientes hospitalizados con multimorbilidades y adultos mayores con pronóstico de vida razonable, en quienes los requerimientos nutricionales no puedan cumplirse por vía oral, se debe administrar nutrición enteral. Por ejemplo: Imposibilidad de ingesta oral por más de tres días o ingesta vía oral cubra menos del $50 \%$ de los requerimientos calórico-proteicos por más de una semana. Si no se alcanzan los requerimientos por vía enteral, se puede recomendar nutrición parenteral periférica.

- En pacientes en ventilación no invasiva (VNI), puede haber dificultad en el inicio de alimentación enteral, porque la sonda puede generar fugas de aire y comprometer la efectividad de la VNI, además de dilatar el estómago por aire. Si no se logra establecer alimentación enteral durante las primeras $48 \mathrm{~h}$ en unidad de cuidados intensivos, se debe considerar nutrición parenteral periférica.

- En pacientes intubados y ventilados se debe iniciar nutrición enteral a través de sonda nasogástrica, si hay elevado riesgo de aspiración utilizar sonda nasoyeyunal. La posición prono no es contraindicación para este tipo de nutrición. También es importante considerar que los pacientes en estado crítico aumentan sus requerimientos proteicos a $1,3 \mathrm{~g} / \mathrm{kg} /$ día.

- El trastorno de deglución postextubación puede prolongarse hasta 21 días, en especial en adultos mayores. Según la evaluación de deglución se debe establecer el tipo de nutrición que se le indicará al paciente:

- Paciente sin disfagia: Dieta entera, con líquidos no espesados.

- Paciente con disfagia a líquidos: Dieta entera y adaptar viscosidad de líquidos según evaluación (viscosidad media de $250 \mathrm{mPa} \cdot \mathrm{s}$ o viscosidad alta de $800 \mathrm{mPa} \cdot \mathrm{s}$ ).

- Paciente con disfagia a sólidos: Adaptar textura de sólidos según evaluación, donde generalmente se indica dieta tipo puré espeso (tipo C) o dieta tipo machacable con tenedor (tipo E).
- Paciente con disfagia severa y signos de alteración de seguridad: Dieta exclusiva vía enteral, ya sea por sonda nasogástrica o nasoyeyunal (riesgo de aspiración alto).

- Si no es posible la vía enteral, iniciar nutrición parenteral periférica hasta lograr colocar una sonda nasoenteral o reestablecer la vía oral.

\subsection{Medidas compensatorias}

Se sugiere realizar las siguientes estrategias de compensación a todos los pacientes con DO y COVID-19 ${ }^{35}$, las cuales se basan en el procedimiento de intervención mínima masiva que fue propuesto como una herramienta costo-efectiva de tratamiento de la DO para prevenir las complicaciones de ésta ${ }^{40}$. La intervención consiste en:

- Postura correcta durante la ingesta, utilizando postura chin-down si es necesario (llevar mentón al pecho durante la deglución $)^{41}$. Utilizar nariceras para oxigenoterapia si el paciente lo requiere.

- Promover la autoalimentación del paciente. Si requiere ayuda para comer, el personal debe usar los elementos de protección personal contra aerosoles por el riesgo de tos durante la ingesta.

- Usar la viscosidad óptima para líquidos, de acuerdo a la evaluación del V-VST acortado, seleccionando líquido, néctar (viscosidad $250 \mathrm{mPa} . \mathrm{s}$ ) o pudding (viscosidad 800 $\mathrm{mPa} \cdot \mathrm{s})$ según corresponda.

- Realizar triple adaptación de los sólidos: Adaptación nutricional (calorías, proteínas y agua), reológica (textura y viscosidad) y organoléptica (visual y presentación del alimento). Se debe seleccionar la textura óptima que el paciente pueda masticar y deglutir según condición física ${ }^{42}$.

- Si se requiere nutrición enteral, se recomienda el uso de sonda nasoenteral y posponer la gastrostomía para evitar la generación de aerosoles al realizar el procedimiento.

- Promover higiene oral. El paciente debe cepillarse los dientes y enjugar su boca después de cada ingesta, si no es autónomo, el aseo debe realizarlo el personal de salud con los elementos de protección personal para aerosoles. Si el riesgo de realizar higiene oral es muy alto, posponer esta medida. 
- Los pacientes con traqueostomía que aún requieran asistencia ventilatoria o ventilación mecánica no deben ser alimentados vía oral.

\subsection{Terapia de rehabilitación}

No están recomendados los tratamientos activos en pacientes con COVID-19 por el riesgo de generación de aerosoles ${ }^{35}$. Esto implica no realizar estimulación sensorial, ni maniobras que puedan generar tos como la deglución supraglótica o que requieran coordinación respiración-deglución como la maniobra de Mendelsohn, o ejercicios que requieran la presencia del fonoaudiólogo/a en la habitación del paciente.

\subsection{Neurorrehabilitación}

Las técnicas de neurorrehabilitación se basan en los principios de la neuroplasticidad, y pueden ser periféricas, centrales o una combinación de ambas. Los tipos de estimulación periférica son la estimulación eléctrica neuromuscular de superficie, la estimulación eléctrica intrafaríngea y la estimulación farmacológica. Los tipos de estimulación central son la estimulación transcraneal magnética repetitiva y la estimulación transcraneal por corriente directa. Estas técnicas se han utilizado principalmente en pacientes con disfagia orofaríngea en el contexto de accidente cerebrovascular, así como en cáncer de cabeza y cuello y enfermedad de Parkinson ${ }^{43}$. No existen reportes de su uso en COVID-19, pero al ser técnicas que no generan aerosoles, podrían tener un rol eventual en la rehabilitación futura de estos pacientes.

\subsection{Intervenciones quirúrgicas}

El momento para realizar una cirugía va a depender de la condición del paciente, de la etiología de su disfagia y del procedimiento en sí. El manejo de la patología neoplásica maligna requiere ser discutido caso a caso. En lugares donde los recursos estén disponibles y se levanten las restricciones a las cirugías electivas, se considera realizar gastrostomías a aquellos pacientes que lo requieran. Si los recursos son limitados, patologías no obstructivas o mínimamente obstructivas como webs cricofaríngeas o divertículos de Zenker pueden posponerse si los pacientes no tienen baja de peso o un riesgo de aspiración evidente $^{30}$.

\section{Conclusión}

La disfagia en el contexto de COVID-19, en especial si es postextubación, requiere medidas proactivas para identificarla y manejarla en el periodo agudo de la enfermedad y realizar un adecuado seguimiento con todas las medidas de protección necesarias. Si no identificamos a tiempo la presencia de disfagia en un paciente convaleciente de una patología pulmonar secundaria a la infección viral y ese paciente presenta alteraciones de la seguridad durante la deglución, las consecuencias de una neumonía aspirativa sobre un pulmón dañado pueden ser devastadoras, aumentando la estadía hospitalaria y la morbimortalidad.

\section{Bibliografía}

1. Kertscher B, Speyer R, Fong E, Georgiou AM, Smith M. Prevalence of oropharyngeal dysphagia in the Netherlands: a telephone survey. Dysphagia 2015;30:114-120. Disponible en https://doi. org/10.1007/s00455-014-9584-z.

2. Roy N, Stemple J, Merrill RM, Thomas L. Dysphagia in the elderly: preliminary evidence of prevalence, risk factors, and socioemotional effects. Ann Otol Rhinol Laryngol 2007;116:858-865. Disponible en https://doi. org/10.1177/000348940711601112.

3. Yang EJ, Kim MH, Lim JY, Paik NJ. Oropharyngeal Dysphagia in a community-based elderly cohort: the korean longitudinal study on health and aging. J Korean Med Sci 2013;28:1534-1539. Disponible en https://doi.org/10.3346/jkms.2013.28.10.1534.

4. Takizawa C, Gemmell E, Kenworthy J, Speyer R. A Systematic Review of the Prevalence of Oropharyngeal Dysphagia in Stroke, Parkinson's Disease, Alzheimer's Disease, Head Injury, and Pneumonia. Dysphagia 2016;31:434-441. Disponible en https://doi.org/10.1007/s00455-016-9695-9.

5. Schefold JC, Berger D, Zürcher P, et al. Dysphagia in Mechanically Ventilated ICU Patients (DYnAMICS): A Prospective Observational Trial. Crit Care Med 2017;45:2061-2069. Disponible en https://doi. org/10.1097/CCM.0000000000002765.

6. Baijens L, Clavé P, Cras P, et al. European Society for Swallowing Disorders - European Union Geriatric Medicine Society white paper: oropharyngeal dysphagia as a geriatric syndrome. Clinical Interventions in Aging 2016;11:1403-1428. 
7. Feng MC, Lin YC, Chang YH, et al. The Mortality and the Risk of Aspiration Pneumonia Related with Dysphagia in Stroke Patients. J Stroke Cerebrovasc Dis 2019;28:1381-1387. Disponible en https://doi. org/10.1016/j.jstrokecerebrovasdis.2019.02.011.

8. Jones E, Speyer R, Kertscher B, Denman D, Swan K, Cordier R. Health-Related Quality of Life and Oropharyngeal Dysphagia: A Systematic Review. Dysphagia 2018;33:141-172. Disponible en https:// doi.org/10.1007/s00455-017-9844-9.

9. Patel DA, Krishnaswami S, Steger E, et al. Economic and survival burden of dysphagia among inpatients in the United States. Dis Esophagus 2018;31:1-7. Disponible en https://doi.org/10.1093/dote/dox131.

10. Nguyen S, Zhu A, Toppen W, et al. Dysphagia after Cardiac Operations Is Associated with Increased Length of Stay and Costs. Am Surg 2016;82:890-893.

11. Bonilha HS, Simpson AN, Ellis C, Mauldin P, MartinHarris B, Simpson K. The one-year attributable cost of post-stroke dysphagia. Dysphagia 2014;29:545-552. Disponible en https://doi.org/10.1007/s00455-0149543-8.

12. Departamento de Epidemiología, Ministerio de Salud de Chile. Informe epidemiológico número 32. Enfermedad por SARS-CoV-2. 10 de julio de 2020.

13. Zheng Z, Peng F, Xu B, et al. Risk factors of critical \& mortal COVID-19 cases: A systematic literature review and meta-analysis. J Infect 2020;S0163-4453:30234-6. Disponible en https://doi. org/10.1016/j.jinf.2020.04.021.

14. Wang L, Wang Y, Ye D, Liu Q. Review of the 2019 novel coronavirus (SARS-CoV-2) based on current evidence. Int J Antimicrob Agents 2020;55:1-7.

15. Mao L, Jin H, Wang M, et al. Neurologic Manifestations of Hospitalized Patients With Coronavirus. JAMA Neurol 2020;77:683-690.

16. Dziewas R, Warnecke T. ICU-related dysphagia. En: Ekberg, Dysphagia: diagnosis and treatment. 2nd edition. 2019:157-64.

17. Hafner G, Neuhuber A, Hirtenfelder S, Schmedler B, Eckel H. Fiberoptic endoscopic evaluation of swallowing in intensive care unit patients. Eur Arch Otorhinolaryngol 2008;265:441-446.

18. Ajemian M, Nirmul G, Anderson M, Zirlen D, Kwasnik E. Routine fiberoptic endoscopic evaluation of swallowing following prolonged intubation: implications for management. Arch Surg 2001;36:434437.

19. Par M, Badovinac A, Plancak D. Oral hygiene is an important factor for prevention of ventilatorassociated pneumonia. Acta Clin Croat 2014;53:72-78.

20. Nagami S, Oku Y, Yagi N, et al. Breathingswallowing discoordination is associated with frequent exacerbations of COPD. BMJ Open Resp Res 2017;4:e000202.

21. Kiekens C, Boldrini P, Andreoli A, Avesani R, Gamna F, Grandi M. Rehabilitation and respiratory management in the acute and early postacute phase. "Instant paper from the field" on rehabilitation answers to the COVID-19 emergency. Eur J Phys Rehabil Med 2020;56:323-326. Disponible en https:// doi.org/ 10.23736/S1973-9087.20.06305-4.

22. Santilli de Lima M, Chiarion F, Medeiros G, Ritto A, Furquim de Andrade C. Preliminary results of a clinical study to evaluate the peprformance and safety of swallowing in critical patients with COVID-19. Clinics 2020;75:e2021.

23. Ai T, Yang Z, Hou H, et al. Correlation of Chest CT and RT-PCR Testing for Coronavirus Disease 2019 (COVID-19) in China: A Report of 1014 Cases. Radiology 2020;296:E32-E40. Disponible en https:// doi.org/10.1148/radiol.2020200642.

24. Wang W, Xu Y, Gao R, et al. Detection of SARSCoV-2 in Different Types of Clinical Specimens. JAMA 2020; 323:1843-1844. Disponible en https:// doi.org/10.1001/jama.2020.3786.

25. World Health Organization. Modes of Transmission of virus causing COVID-19: Implications for IPC precaution recomendations 2020,Vols. WHO/2019nCoV/Sci_Brief/Transmission_modes/2020.2, July 9th, 2020.

26. Bourouiba L. Turbulent Gas Clouds and Respiratory Pathogen Emissions: Potential Implications for Reducing Transmission of COVID-19. JAMA. 2020;10.1001/jama.2020.4756. Disponible en https:// doi.org/10.1001/jama.2020.4756.

27. Bolton L, Mills C, Wallace S, Brady MC; Royal College of Speech and Language Therapists (RCSLT) COVID-19 Advisory Group. Aerosol generating procedures, dysphagia assessment and COVID-19: A rapid review. Int J Lang Commun Disord 2020;10.1111/1460-6984.12544. Disponible en https://doi.org/10.1111/1460-6984.12544.

28. ASHA Association. ASHA guidance to SLPs regarding aerosol generating procedures. https://www.asha.org/ SLP/healthcare, 2020.

29. Mattei A, Amy de la Bretèque B, Crestani S, et al. Guidelines of clinical practice for the management of swallowing disorders and recent dysphonia in the context of the COVID-19 pandemic. Eur Ann Otorhinolaryngol Head Neck Dis 2020; 137:173175. Disponible en https://doi.org/10.1016/j. anorl.2020.04.011.

30. Fritz MA, Howell RJ, Brodsky MB, et al. Moving Forward with Dysphagia Care: Implementing Strategies during the COVID-19 Pandemic and Beyond. Dysphagia 2020;1-9. Disponible en https:// doi.org/10.1007/s00455-020-10144-9.

31. Burgos R, Sarto B, Segurola H, et al. Traducción y validación de la versión en español de la escala EAT10 (Eating Assessment Tool-10) para el despistaje de la disfagia [Translation and validation of the Spanish version of the EAT-10 (Eating Assessment Tool-10) for the screening of dysphagia]. Nutr 
Hosp 2012;27:2048-2054. Disponible en https://doi. org/10.3305/nh.2012.27.6.6100.

32. Fernández-Rosati J, Lera L, Fuentes-López E, Albala C. Validez y confiabilidad del cuestionario Eating Assessment Tool 10 (EAT-10) para detectar disfagia en adultos mayores chilenos [Validation of the eat-10 score to detect dysphagia in older people]. Rev Med Chile 2018;146:1008-1015. Disponible en https://doi. org/10.4067/s0034-98872018000901008.

33. Salassa JR. A functional outcome swallowing scale for staging oropharyngeal dysphagia. Dig Dis 1999;17:230-234. Disponible en https://doi. org/10.1159/000016941.

34. Clavé P, Arreola V, Romea M, Medina L, Palomera E, Serra-Prat M. Accuracy of the volume-viscosity swallow test for clinical screening of oropharyngeal dysphagia and aspiration. Clin Nutr 2008;27:806815. Disponible en https://doi.org/10.1016/j. clnu.2008.06.011.

35. Clavé P, Arreola V, Martín A, et al. Procedimientos básicos para evaluar y tratar la dIsfagia orofaríngea en pcientes con infección por COVID-19. En: Guía práctica del Hospital de Mataró, Catalunya, España, Mataró, 2020.

36. Clavé $P$. 10 basic procedures to assess and treat oropharyngeal dysphagia in patients with COVID-19 (SARS-CoV-2) infection at Hospital de Mataró. Expert opinion practical guidance from Hospital de Mataró, Catalonia, Spain, vol. Webinar ESSD, 2020.

37. Béchet S, Hill F, Gilheaney Ó, Walshe M.

Diagnostic Accuracy of the Modified Evan's Blue Dye Test in Detecting Aspiration in Patients with Tracheostomy: A Systematic Review of the Evidence. Dysphagia 2016;31:721-9. Disponible en https://doi. org/10.1007/s00455-016-9737-3.

38. Zou L, Ruan F, Huang M, et al. SARS-CoV-2 Viral Load in Upper Respiratory Specimens of Infected Patients. N Engl J Med 2020;382:11771179. Disponible en https://doi.org/10.1056/ NEJMc2001737.

39. Barazzoni R, Bischoff SC, Breda J, et al. ESPEN expert statements and practical guidance for nutritional management of individuals with SARS-CoV-2 infection. Clin Nutr 2020;39:1631-1638. Disponible en https://doi.org/10.1016/j.clnu.2020.03.022.

40. Martín A, Ortega O, Roca M, Arús M, Clavé P. Effect of A Minimal-Massive Intervention in Hospitalized Older Patients with Oropharyngeal Dysphagia: A Proof of Concept Study. J Nutr Health Aging 2018;22:739-747. Disponible en https://doi. org/10.1007/s12603-018-1043-3.

41. Leigh JH, Oh BM, Seo HG, et al. Influence of the chin-down and chin-tuck maneuver on the swallowing kinematics of healthy adults. Dysphagia 2015;30:89-98. Disponible en https://doi.org/10.1007/ s00455-014-9580-3

42. Costa A, Carrión S, Puig-Pey M, Juárez F, Clavé P. Triple Adaptation of the Mediterranean Diet: Design of A Meal Plan for Older People with Oropharyngeal Dysphagia Based on Home Cooking. Nutrients 2019;11:425. Disponible en https://doi.org/10.3390/ nu11020425.

43. Cabib C, Ortega O, Kumru H, et al. Neurorehabilitation strategies for poststroke oropharyngeal dysphagia: from compensation to the recovery of swallowing function. Ann N Y Acad Sci 2016;1380:121-138. Disponible en https://doi. org/10.1111/nyas. 13135 . 